\title{
PROCEDIMENTOS PARA IMPLANTAÇÃO DE UM LABORATÓRIO DE ESPECTROMETRIA GAMA NO LABORATÓRIO DE INSTRUMENTAÇÃO NUCLEAR (LDIN) DO INSTITUTO MILITAR DE ENGENHARIA (IME).
}

\author{
Jean Carlos Barbosa da Silva \\ Instituto Militar de Engenharia \\ Praça General Tibúrcio, 80, Praia Vermelha. Urca - Rio de Janeiro \\ jcbds5@yahoo.com.br \\ Gabriela Martins Duarte \\ Instituto Militar de Engenharia \\ Praça General Tibúrcio, 80, Praia Vermelha. Urca - Rio de Janeiro \\ gabrimduarte92@gmail.com
}

\begin{abstract}
RESUMO
Espectrometria e espectroscopia são técnicas utilizadas para a obtenção de informações relativas a uma fonte radioativa conhecida ou desconhecida. A espectrometria lida com a medida da atividade de fontes radioativas, já a espectroscopia caracteriza e identifica essas fontes, através do padrão espectral delas. A versatilidade da espectrometria permite sua aplicação em várias áreas de conhecimento, tais como indústrias, centros de pesquisa, monitoramento ambiental, entre outras. Conhecendo os parâmetros e características da espectrometria, este trabalho tem como objetivo determinar as melhores condições para a implantação de um Laboratório de Espectrometria gama no Laboratório de Instrumentação Nuclear (LDIN) do Instituto Militar de Engenharia (IME).
\end{abstract}

Palavra-chave: Espectrometria; Espectroscopia; Padrão espectral; Cintiladores; Semicondutores.

\section{ABSTRACT}

Spectrometry and spectroscopy are techniques used to use information from a known or unknown radioactive source. A spectrometer with activity measurement of radioactive sources, already with spectroscopy, identifies and identifies these sources by their spectral pattern. A versatility of spectrometry allows its application in various areas of knowledge, such as industries, research centers, environmental monitoring, among others. Knowing the parameters and characteristics of spectrometry, this work aims to determine the best conditions for the implementation of a Gamma Spectrometry Laboratory at the Nuclear Instrumentation Laboratory (LDIN) of the Military Institute of Engineering (IME).

Keywords: Spectrometry; Spectroscopy; Spectral pattern; Scintillators; Semiconductors.

\section{Como Citar:}

SILVA, Jean Carlos Barbosa da; DUARTE, Gabriela Martins. Procedimentos para Implementação de um Laboratório de Espectrometria Gama no Laboratório de Instrumentação Nuclear (LDIN) do Instituto Militar de Engenharia. In: SIMPÓSIO DE 
PESQUISA OPERACIONAL E LOGÍSTICA DA MARINHA, 19., 2019, Rio de Janeiro, RJ. Anais [...]. Rio de Janeiro: Centro de Análises de Sistemas Navais, 2019.

\section{INTRODUÇÃO}

A espectrometria, assim como a espectroscopia, são técnicas utilizadas para se obter informações relativas a uma fonte radioativa conhecida ou desconhecida. Enquanto a espectrometria trabalha fundamentalmente com a medida da atividade de fontes radioativas, o enfoque da espectroscopia é caracterização e identificação dessas fontes, através do padrão espectral das mesmas. A versatilidade da espectrometria permite sua aplicação em várias áreas de conhecimento, tais como indústrias, centros de pesquisa, monitoramento ambiental, entre outras. Devido à resolução em energia associada aos tipos de detectores, geralmente utilizados em espectrometria, pode-se obter uma melhor distinção dos elementos radioativos presentes na amostra analisada. Na visualização da Gaussiana, no software de análise de espectro da amostra, observa-se a ocorrência do efeito fotoelétrico no material do detector (fotopico), o que permite discriminar as diversas energias detectadas no equipamento.

Outra característica analisada é o tempo morto na ordem de nanosegundos, fato este associado à melhor rapidez na obtenção dos espectros. Vale ressaltar ainda, que se trata de um ensaio não destrutivo e, portanto, a amostra não necessita de nenhum tratamento químico.

Tratando-se de espectrometria gama, os detectores mais utilizados são os do tipo semicondutores e os cintiladores inorgânicos. Para garantir bom desempenho do detector, na aplicação desta técnica, alguns parâmetros necessitam de certa atenção:

(i) Calibração do sistema de medida;

(ii) Resolução do fotopico;

(iii) Baixa energia mínima detectável (MDA);

(iv) O uso de fontes gama certificadas;

(v) Eficiência de contagens por energia de fótons.

Conhecendo os parâmetros e características da espectrometria, este trabalho tem como objetivo determinar as melhores condições para a implantação de um Laboratório de Espectrometria gama no Laboratório de Instrumentação Nuclear (LDIN) do Instituto Militar de Engenharia (IME).

\section{REVISÃO BIBLIOGRÁFICA}

Em um trabalho que está em elaboração determinou-se a eficiência relativa de um detector de germânio hiperpuro (HPGe) em relação a um detector cintilador de iodeto de sódio ativado a tálio $\mathrm{NaI}(\mathrm{Tl})$. Na contagem do experimento, detectou-se a presença de Tálio208 (radionuclídeo proveniente do decaimento do Tório-232), devido à proximidade do Morro da Babilônia (no bairro da Urca, na cidade do Rio de Janeiro) ao laboratório. Diante disso, constatou-se que para bom desempenho no experimento é necessário que haja blindagem adequada no aparato experimental, para assim, reduzir a influência do BG (background) na contagem do experimento.

Após atenuar a radiação do BG, monitoram-se as tensões e o aterramento existente nas instalações do IME, uma vez que estes fatores podem conturbar as medições coletadas pelos detectores. Caso essas medidas não sejam tomadas, é possível que haja discrepância nas estatísticas obtidas e, consequentemente, um sistema mal calibrado. 


\section{METODOLOGIA}

O experimento utilizou fontes radioativas de Eu-152 e Co-60 para realizar a calibração do aparato experimental, obedecendo-se três diretrizes principais: (i) rastreabilidade dos materiais utilizados, visando garantir a confiabilidade das medidas por meio da calibração e da manutenção do aparato experimental; (ii) monitoramento de variações na rede elétrica e aterramento, uma vez estas variações podem originar desvios nas medidas; (iii) excelência na blindagem do aparato experimental para garantir que demais fontes radioativas, presentes no local, não interfiram no experimento.

Foram ajustados os ganhos para detecção com o $\mathrm{NaI}(\mathrm{Tl})$ no amplificador para ganho grosso 2 e ganho fino 3.5 e no pré-amplificador foi utilizada a capacitância de $100 \mathrm{pF}$. Para a detecção utilizando o HPGe foi respeitado a mesma configuração geométrica e, o ganho foi ajustado por tentativa e erro para que a maior energia do Co-60 coincidisse no canal similar ao do detector $\mathrm{NaI}(\mathrm{Tl})$. As tensões da alta tensão para o HPGe e para o $\mathrm{NaI}(\mathrm{Tl})$ foram $800 \mathrm{~V}$ e $2300 \mathrm{~V}$, respectivamente. Os manuais de todos os fabricantes foram consultados, antes da montagem do aparato experimental, a fim de se obter excelência nas medidas obtidas.

\section{RESULTADOS}

Os resultados obtidos serão demonstrados a seguir, e discutidos no tópico CONCLUSÃO.

Apresentou-se, inicialmente, na Figura 1 a reta de calibração do detector semicondutor HPGe, levantada utilizando a fonte de Eu-152 nas seguintes energias (keV): 244,$69 ; 344,28 ; 411,12 ; 443,96$; 586,27; 688,67; 778,9; 867,38; 964,08; 1085,84; 1112,08; 1212,95 e 1408,01 . Com a finalidade de verificar a validação da reta de calibração fez-se medidas utilizando a fonte de Co-60 existente no laboratório, conforme mostrado na Figura 2.

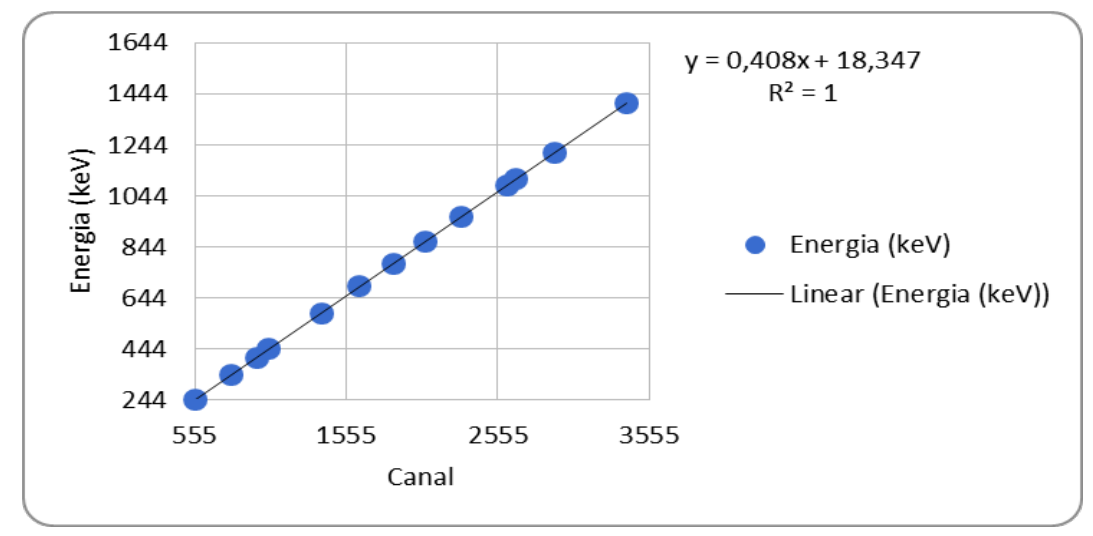

Figura 1 - Reta de calibração do detector HPGe. 


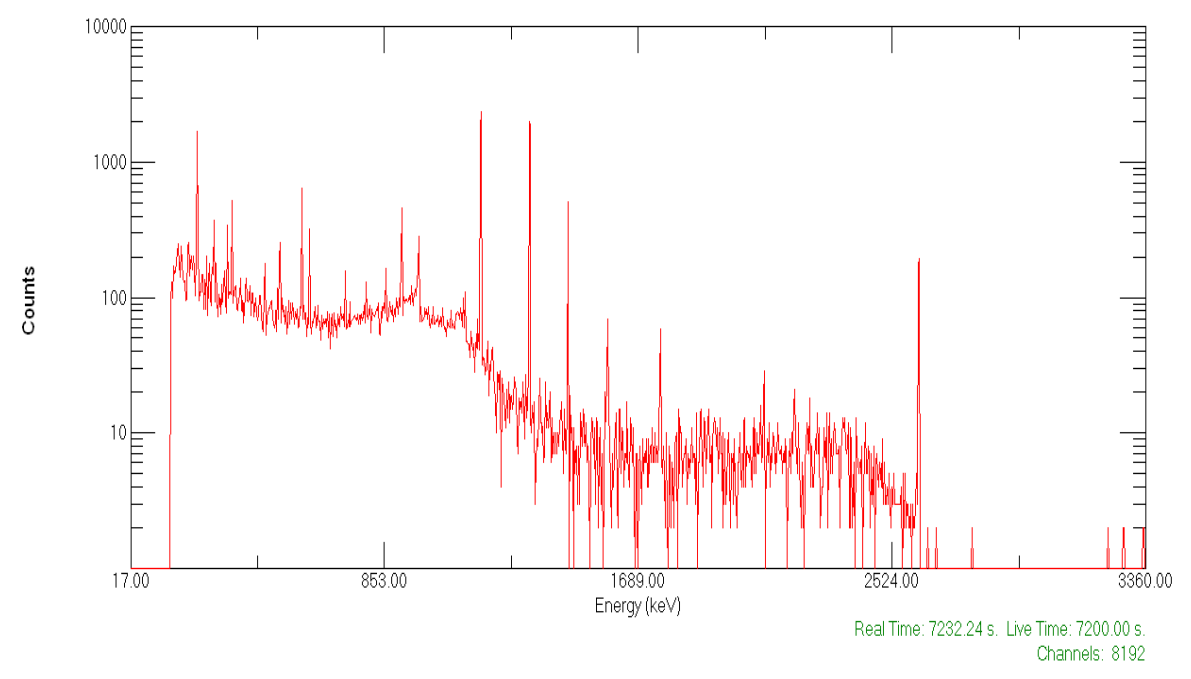

Figura 2 - Espectro líquido obtido com a fonte de Cobalto-60 no detector HPGe.

Em seguida, serão apresentados os resultados obtidos nas medidas com o detector cintilador $\mathrm{NaI}(\mathrm{Tl})$. A Figura 3 exibe a reta de calibração com a fonte de Eu-152 nas respectivas energias (keV): 344,28; 778,9; 964,08; 1089,74; 1408,01. Seguido da Figura 4 que expõe o espectro obtido com a fonte de Co-60 utilizada no detector $\mathrm{NaI}(\mathrm{Tl})$, com a mesma finalidade aplicada no detector HPGe.

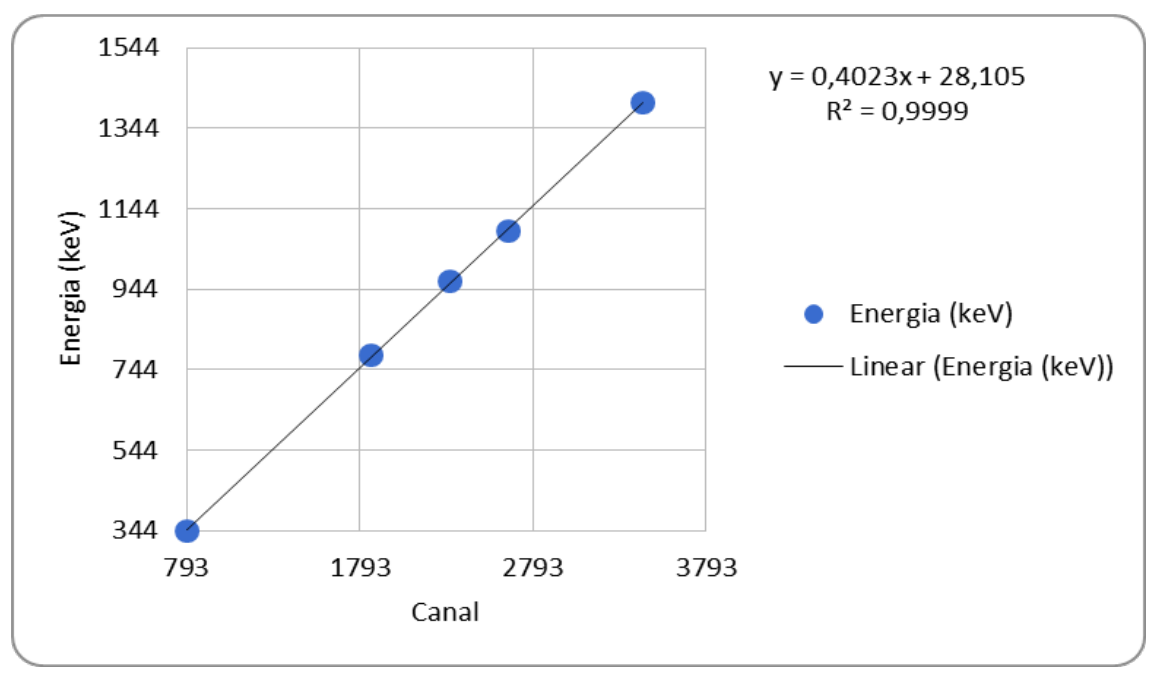

Figura 3 - Reta de calibração do detector $\mathrm{NaI}(\mathrm{Tl})$. 


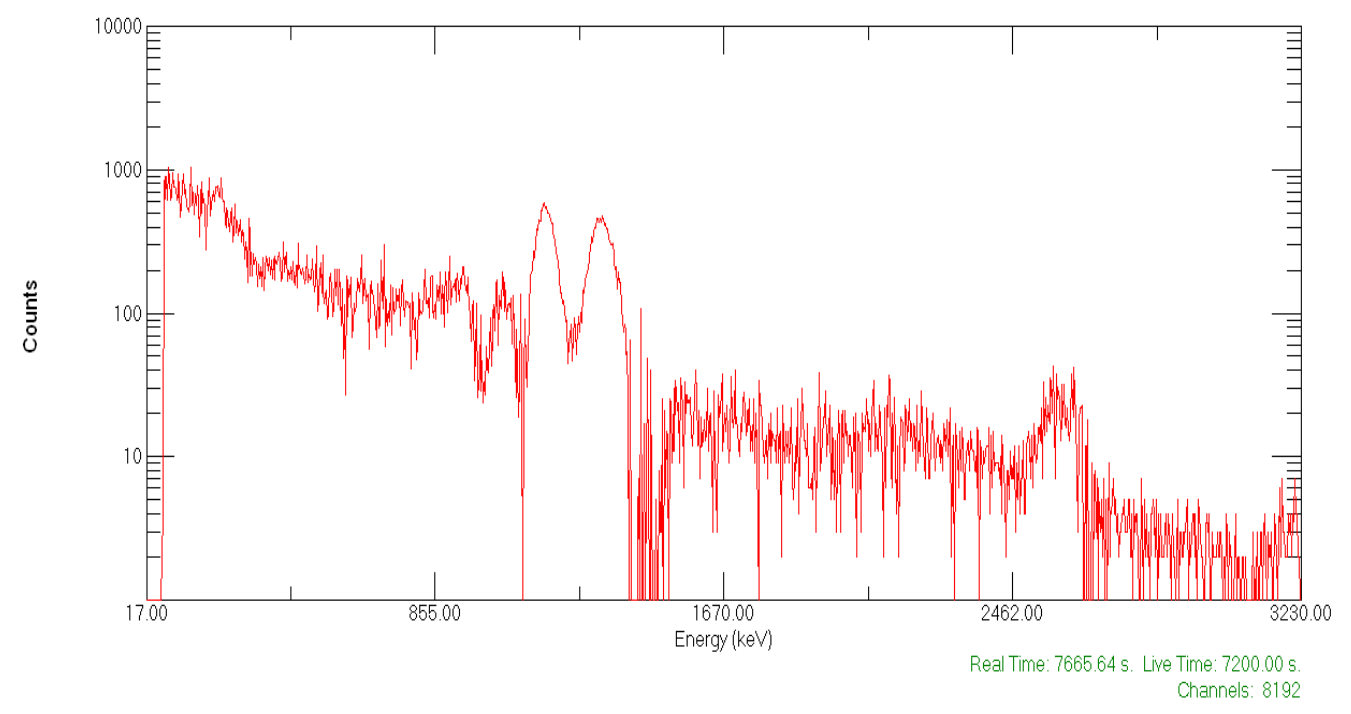

Figura 4 - Espectro líquido obtido com fonte de Cobalto-60 no detector $\mathrm{NaI}(\mathrm{Tl})$.

\section{CONCLUSÕES}

Inicialmente, obteve-se a reta de calibração para o aparato experimental do HPGe e do $\mathrm{NaI}(\mathrm{Tl})$, conforme mostrado na Figura 1 e Figura 3, respectivamente.

Após a realização da calibração, com a finalidade de validar a reta de calibração dos equipamentos, com o auxílio de uma fonte de Co-60 de $41 \mathrm{kBq}$, obteve-se as energias $1171,91 \mathrm{keV}$ e $1328,74 \mathrm{keV}$ no detector $\mathrm{NaI}(\mathrm{Tl})$ e $1171,72 \mathrm{keV}$ e1331,66keV no HPGe, próximos aos valores esperados $1173,23 \mathrm{keV}$ e $1332,49 \mathrm{keV}$ conforme disponível no LaraWeb. Confirmando que a calibração foi satisfatória. Obteve-se, também, a energia $2613,62 \mathrm{keV}$ do Tl-208 no detector HPGe, também próxima do esperado, de acordo com o LaraWeb, 2614,51keV. Entretanto, no $\mathrm{NaI}(\mathrm{Tl})$ o pico de energia não é bem definido pelo fato de sua resolução não ser tão boa e, a radiação ser proveniente do BG. Estes resultados foram apresentados, na Figura 2 para o HPGe e Figura 4 para o NaI(Tl). Além disso, a partir das análises dos espectros obtidos, constatou-se que:

- a blindagem ao redor do aparato experimental privilegia a discriminação de energia dos espectros levantados, confirmando a boa geometria das condições experimentais utilizadas;

observou-se a redução da influência das energias provenientes do espalhamento Compton no detector, proveniente do radionuclídio Tálio-208, existente no espectro de radiação de fundo do laboratório de medida;

- $\quad$ durante a realização dos experimentos e sua posterior análise, notou-se um deslocamento do espectro obtido ao longo do tempo de realização, quando todas as condições do experimento foram mantidas. Suspeitou-se então da qualidade de fornecimento de energia elétrica do laboratório. 
mediu-se então as tensões entre: fases, fase neutro, fase terra, terra-neutro e, ao longo do dia, mediu-se variações de até 47V entre as medidas de tensão terra-neutro da instalação. Essas discrepâncias podem se sobrepor às energias de interesse, gerando estatísticas menos confiáveis e deslocamento de espectro medido. O problema foi atribuído ao sistema de aterramento do prédio e está sendo solucionado.

Imediatamente após a análise descrita acima, a implantação do laboratório de espectrometria gama foi interrompida. A metodologia proposta será refeita após o reparo no sistema de aterramento, para assim, dar continuidade à implantação do mesmo.

\section{REFERÊNCIAS}

[1] KNOLL, Glenn F. Radiation Detection and Measurements. 3 ed. New York: John Wiley \& Sons Inc, 1989. 816 p. ISBN 0-417-07338-5.

[2] TAUHATA, Luiz, SALATI, Ivan, PRINZIO, Renato Di, PRINZIO, Antonieta R. Di. Radioproteção e Dosimetria: Fundamentos - 9 rev. Rio de Janeiro: Instituto de Radioproteção e Dosimetria/Comissão Nacional de Energia Nuclear, 2013, 345 p. 